\title{
Performance Comparison of Time Delay Estimation for Whole and Dispersed Spectrum Utilization in Cognitive Radio Systems
}

\author{
Hasari Celebi and Khalid A. Qaraqe \\ Department of Electrical and Computer Engineering \\ Texas A\&M University at Qatar \\ PO Box 23874 Texas A\&M Engineering Building \\ Education City, Doha, Qatar \\ \{hasari.celebi,khalid.qaraqe\}@qatar.tamu.edu
}

\author{
Huseyin Arslan \\ Electrical Engineering Department \\ University of South Florida \\ 4202 E. Fowler Avenue, ENB-118 \\ Tampa, FL, 33620 \\ arslan@eng.usf.edu
}

\begin{abstract}
In cognitive radio systems, information is transmitted and received over multiple dispersed bands using dispersed spectrum utilization approach whereas information is transmitted and received over a single band using whole spectrum utilization approach. In this paper, performance of both approaches are compared theoretically considering CRLB and maximum likelihood (ML) method for time delay estimation. The results show that dispersed spectrum utilization methods have a great potential to exploit the efficiency of spectrum utilization and support goal driven and autonomous cognitive radio systems.
\end{abstract}

\section{INTRODUCTION}

Cognitive radio (CR) is a novel approach for developing intelligent and sophisticated wireless systems [1], [2], which can require utilization of spectrum resources dynamically [3], [4]. CR has a capability to exploit the spectrum utilization due to its spectrum agility. The available spectrum can be mainly in two forms; single band, i.e. whole spectrum, and multiband, i.e. dispersed spectrum [5]-[8] ${ }^{1}$. In the whole spectrum utilization approach, the transmit signal for a single CR user occupies a single band as illustrated in Fig. 1a, whereas, in the dispersed spectrum utilization approach, it occupies multiple bands (e.g. $K$ number of adjacent or nonadjacent bands) simultaneously as shown in Fig. 1b. Note that dispersed spectrum utilization is a method that realizes frequency diversity in full extent in wireless systems.

Since whole spectrum utilization is the conventional way of transmitting signal numerous technologies and transceiver architectures have been developed for such approach. For instance, cognitive positioning systems (CPSs) along with fundamental limits of time delay estimation are studied considering whole spectrum utilization approach in [4], [13].

The dispersed spectrum utilization approach is first discussed briefly in [5] and investigated with details in [6]. In [6], a CR receiver architecture for the dispersed spectrum utilization based on the idea of processing the receive signal

\footnotetext{
${ }^{1}$ Note that another way to implement dispersed spectrum utilization approach is to employ orthogonal frequency division multiplexing (OFDM) technology, where the sub-carriers corresponding to the used and unused bands are activated and nulled, respectively [6], [9]-[12]. However, such approach is not considered in this study.
}

at multiple branches is proposed. Each branch considers one available band, and down-converts the signal according to the center frequency of that band. By this way, signals with narrower bandwidths can be processed at each branch. As a result, investigation on performance comparison of whole and dispersed spectrum utilization is of considerable interest for development of goal driven and autonomous feature of CR systems. Hence, in this paper, the performance of whole and dispersed spectrum utilization methods are compared theoretically considering time delay estimation problem in CR systems. Exact and approximate Cramer-Rao lower bound (CRLB) over AWGN channel for both approaches are presented. Then, performance comparison of both approaches is carried out based on exact and approximate CRLBs and optimal Maximum Likelihood (ML) method for time delay estimation through computer simulations.

The paper is organized as follows. In Section II, the system model for whole and spectrum utilization approaches are provided. In Section III, ML, and exact and approximate CRLBs of time delay estimate for both approaches are presented. In Section IV, results and discussions are provided. Finally, the conclusions are presented in Section V.

\section{SySTEM MODEL}

To the best of author's knowledge, there is not any solid study on channel behavior of dispersed spectrum utilization method in the literature. In practice, the observed channel at each band can be different depending on how dispersed bands are located in the spectrum. As a result, for the sake of simplifying the performance comparison analysis and exploring the fundamental considerations, we perform analysis of both approaches considering AWGN channel in this study. In addition, we assume that all the dispersed bands experience different AWGN channels. In the following sections, system models for both approaches are presented.

\section{A. Whole Spectrum Utilization}

The system model shown in Fig. 2 is considered for whole spectrum utilization in this paper. The baseband transmit signal 


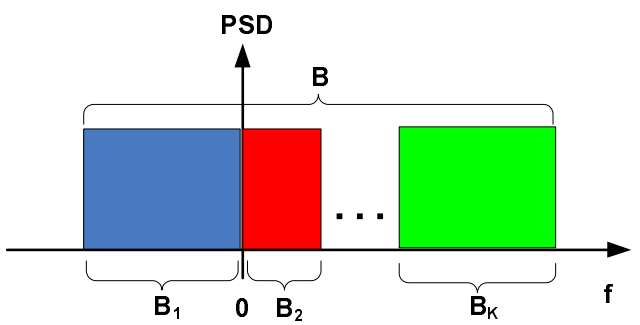

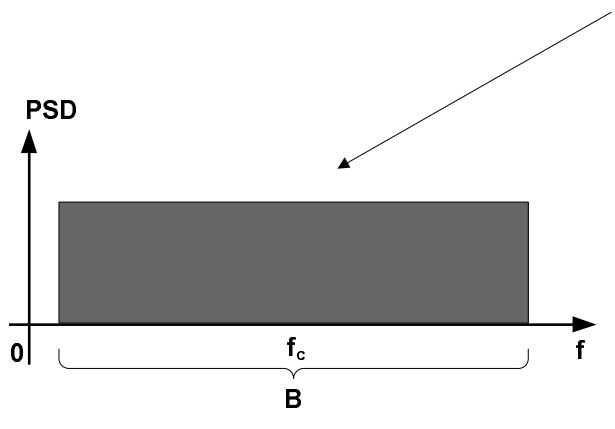

a) Whole Spectrum Utilization

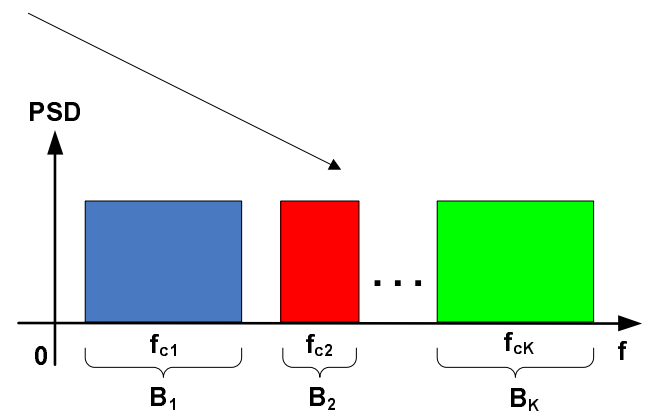

b) Dispersed Spectrum Utilization

Fig. 1. Illustration of whole and dispersed spectrum utilization in cognitive radio systems.

$s(t)$ with absolute bandwidth of $B([-\mathrm{B} / 2, \mathrm{~B} / 2])$ that occupies a whole band shown in Fig. 1a is given by

$$
s(t)=\sum_{l} d_{l} p\left(t-l T_{s}\right),
$$

where $d_{l}$ is the real data for $l$ th symbol, $p(t)$ is the pulse signal with energy $E_{p}$ and duration $T_{p}$, i.e., $p(t)=0$ for $t \notin\left[0, T_{p}\right]$ and $T_{s}$ is the symbol duration. The baseband signal $s(t)$ is transmitted over AWGN channel and the corresponding baseband representation of receive signal $r(t)$ is given by

$$
r(t)=\alpha s(t-\tau)+n(t),
$$

where $\alpha$ and $\tau$ are the path coefficient and delay, respectively, and $n(t)$ is independent white Gaussian noise with spectral density of $\sigma^{2}$. At the receiver side, $r(t)$ is used to perform ML time delay estimation.

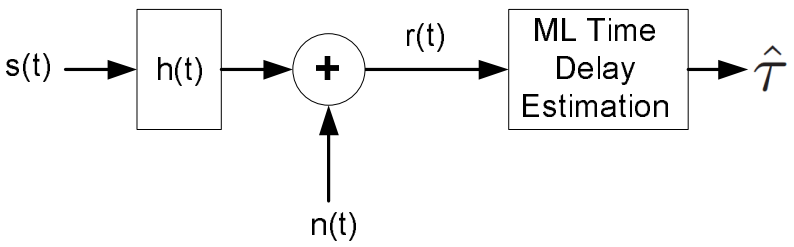

Fig. 2. System model for whole spectrum utilization.

\section{B. Dispersed Spectrum Utilization}

The system model shown in Fig. 3 is considered for dispersed spectrum utilization approach in this paper. The baseband transmit signals $s_{i}(t)$ at $i$ th branch $(i=1, \ldots, K)$ with corresponding absolute bandwidth of $B_{i}$ that occupies dispersed band shown in Fig. 1b is given by

$$
s_{i}(t)=\sum_{l} d_{i, l} p_{i}\left(t-l T_{s}\right),
$$

where $d_{i, l}$ is the real data for the $l$ th symbol of signal $i$, and $p_{i}(t)$ represents a pulse with energy $E_{p i}$ and duration $T_{p i}$, i.e., $p_{i}(t)=0$ for $t \notin\left[0, T_{p i}\right]$. The baseband signal $s_{i}(t)$ is transmitted and assuming that each signal experience different AWGN channel and the corresponding baseband representation of receive signal at $i$ th branch $r_{i}(t)$ is given by

$$
r_{i}(t)=\alpha_{i} s_{i}(t-\tau)+n_{i}(t)
$$

where $\alpha_{i}$ and $\tau$ are the path coefficient and delay for $i$ th branch, respectively, and $n_{i}(t)$ is independent white Gaussian noise with spectral density of $\sigma_{i}^{2}$. At the receiver side, $r_{i}(t)$ at $i$ th branch is used to perform ML time delay estimation. For the fair comparison, we assume $d_{i, l}=d_{l}$, which implies that $d_{l}$ data in case of whole band is transmitted over each dispersed band.

\section{Performance Analysis}

In this section, ML, and exact and approximate CRLB expressions of time delay estimate for both whole and dispersed spectrum utilization are presented.

\section{A. Whole Spectrum Utilization}

Let $\boldsymbol{\theta}=[\tau]$ represent the vector of unknown signal parameters, where $\alpha$ is assumed to be known. The observation interval $[0, T]$ is considered and it can be expressed as $T=N T_{s}$, where $N$ is the number of observation symbol. Then, the ML 


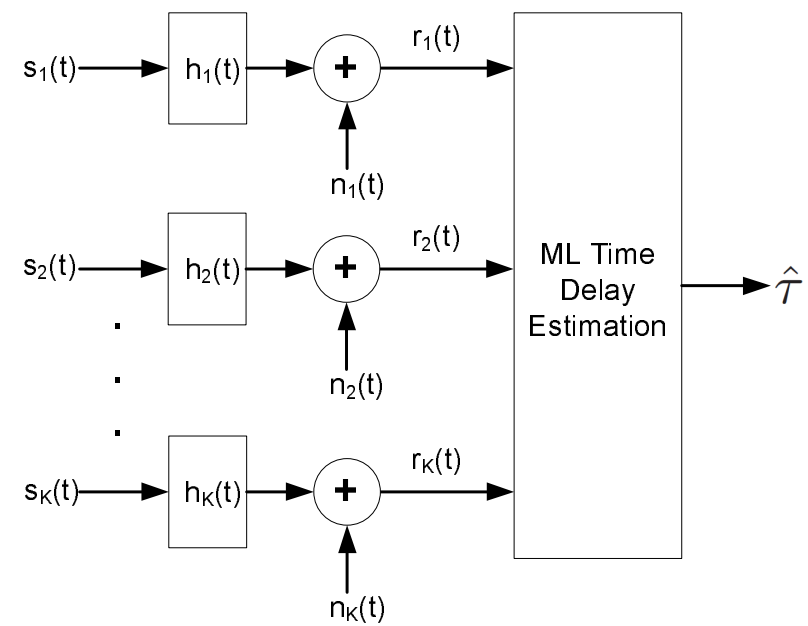

Fig. 3. System model for the dispersed spectrum utilization.

estimate for $\boldsymbol{\theta}$ is given by [14]

$$
\hat{\boldsymbol{\theta}}_{\mathrm{ML}} \approx \arg \max _{\boldsymbol{\theta}}\left\{\frac{1}{\sigma^{2}} \int_{0}^{T} \alpha r(t) s(t-\tau) \mathrm{d} t\right\},
$$

where $E=\int_{0}^{T}[s(t-\tau)]^{2} \mathrm{~d} t$ is the signal energy. Then, the CRLB for unbiased time delay estimators is given by [14]

$$
\mathrm{CRLB}=\frac{1}{\gamma \tilde{E}} .
$$

where $\gamma=\alpha^{2} / \sigma^{2}$ and $\tilde{E}=\int_{0}^{T}\left[s^{\prime}(t-\tau)\right]^{2} \mathrm{~d} t$. Assuming that the spectral density of $p(t)$ is constant over the $B$, then the approximate CRLB expression is given by [14]

$$
\mathrm{CRLB}_{w h}=\frac{1}{\frac{4 \pi^{2}}{3} \mathrm{SNR} B^{2}}
$$

where SNR is defined as

$$
\mathrm{SNR}=\frac{\alpha^{2} N d_{l}^{2} E_{p}}{\sigma^{2}} .
$$

\section{B. Dispersed Spectrum Utilization}

Similarly, let $\boldsymbol{\theta}=[\tau]$ represents the vector of unknown signal parameters, where $\alpha_{i}$ are assumed to be known. The observation interval $T_{i}$ at $i$ th branch can be expressed as $T_{i}=N_{i} T_{s}$, where $N_{i}$ is considered for an integer $N_{i}$ for $i=1, \ldots, K$. Then, the ML estimate for $\boldsymbol{\theta}$ is given by

$$
\hat{\boldsymbol{\theta}}_{\mathrm{ML}} \approx \arg \max _{\boldsymbol{\theta}}\left\{\sum_{i=1}^{K} \frac{1}{\sigma_{i}^{2}} \int_{0}^{T} \alpha_{i} r_{i}(t) s_{i}(t-\tau) \mathrm{d} t\right\} .
$$

The exact CRLB for dispersed spectrum utilization systems is derived in [6], which has the following form,

$$
\operatorname{CRLB}_{\text {disp }}=\frac{1}{\sum_{i=1}^{K} \gamma_{i} \tilde{E}_{i}} .
$$

where $\gamma_{i}=\alpha_{i}^{2} / \sigma_{i}^{2}$ and $\tilde{E}_{i}=\int_{0}^{T}\left[s_{i}^{\prime}(t-\tau)\right]^{2} \mathrm{~d} t$. Assuming that the spectral density of $p(t)$ is constant over the $B$, then the approximate CRLB expression is given by [14]

$$
\mathrm{CRLB}_{\text {disp }}=\frac{1}{\frac{4 \pi^{2}}{3} \sum_{i=1}^{K} \mathrm{SNR}_{i} B_{i}^{2}},
$$

where $\mathrm{SNR}_{i}$ is defined as

$$
\mathrm{SNR}_{i}=\frac{\alpha_{i}^{2} N_{i} d_{l}^{2} E_{p_{i}}}{\sigma_{i}^{2}} .
$$

By examining (7) and (11), the performance comparison of whole and dispersed spectrum utilization in terms of CRLB depends on the values of SNR $B, \mathrm{SNR}_{i}$, and $B_{i}$. The following are three possible cases for the CRLB performance comparison of both approaches,

$$
\begin{cases}\mathrm{CRLB}_{\text {disp }}>\mathrm{CRLB}_{w h}, & \text { If } \sum_{i=1}^{K} \mathrm{SNR}_{i} B_{i}^{2}<\mathrm{SNR} B^{2}, \\ \mathrm{CRLB}_{\text {disp }}<\mathrm{CRLB}_{w h}, & \text { If } \sum_{i=1}^{K} \mathrm{SNR}_{i} B_{i}^{2}>\mathrm{SNR}^{2}, \\ \mathrm{CRLB}_{\text {disp }}=\mathrm{CRLB}_{w h}, & \text { If } \sum_{i=1}^{K} \mathrm{SNR}_{i} B_{i}^{2}=\mathrm{SNR} B^{2} .\end{cases}
$$

From the third condition in (13), it can be observed that the same CRLB can be achieved theoretically by selecting the appropriate SNR levels and absolute bandwidths $B$ for both technique. Some representative applications of (13) are given as follows. This set of equation is useful for the selection of the $K, S N R_{i}, B_{i}$ parameters in dispersed spectrum utilization techniques that can provide the same performance as whole spectrum utilization techniques with $S N R$ and $B$ parameters. It also can be useful to quantify the equivalent of $K$ number of dispersed bandwidth $B_{i}$ as a whole bandwidth $B$ for given $S N R_{i}$ and $S N R$, respectively. Finally, the conditions in (13) is useful for the optimization mechanism in range accuracy adaptation [5], [6], [13] which is a feature of the CPSs. During the optimization of spectrum parameters, range accuracy adaptation algorithm can select the optimal spectrum parameters (e.g. $K, B, S N R$ ) using the conditions in (13).

\section{RESUlts}

In this section, performance of both exact and approximate CRLBs are compared considering whole spectrum utilization systems. This is followed by performance comparison of both whole and dispersed spectrum utilization approaches considering the approximate CRLB and ML method.

The following simulation parameters are used for comparing the exact and approximate CRLBs. Note that these bounds are also compared to the performance of ML. For the pulse shape, the following Gaussian second order derivative pulse shape is employed

$$
p(t)=A\left(1-\frac{4 \pi t^{2}}{\zeta^{2}}\right) e^{-2 \pi t^{2} / \zeta^{2}},
$$

where $A$ and $\zeta$ are parameters that are used to adjust the pulse energy and the pulse width, respectively. $A$ is selected in order to generate pulses with unit energy. For the given pulse shape, pulse width is defined as $T_{p}=2.5 \zeta$ [6], where $\zeta=1 / B$ and $B=1 \mathrm{MHz}$. Moreover, the symbol duration of $T_{\text {sym }}=10 \zeta$ is employed. Uniformly distributed delay $T_{a}=0.8 \zeta$ is considered, i.e. $U\left[0, T_{a}\right]$. The number of training 
symbol $N$ that is considered is 1 and $d_{l}=[1]$. The results are obtained over 3000 channel realizations and plotted in Fig. 4. According to the results, the exact CRLB performs better than the approximate CRLB by approximately $3.7 \mathrm{~dB}$. This is due to the flat spectrum assumption made during the derivation of approximate CRLB.

ML method can be implemented by cross-correlating the received signal with template signal and computing the minimum square error (MSE) of time delay estimates. The time delay estimates are subject to ambiguity errors caused by the oscillatory nature of the signal correlation function [15]. The results in Fig. 4 as well as the results in the literature [15] indicates that MSE is a function of SNR exhibiting threshold phenomenon. The SNR threshold for the results in Fig. 4 is $16 \mathrm{~dB}$ and it divides the SNR region into two distinct regimes [15], which are low and medium SNR regions.

Note that the SNR point where the transition from low to medium SNR regimes occurs is referred as SNR threshold. MSE demonstrates a nonlinear behavior in the low SNR regime, whereas this behavior becomes linear after the SNR threshold. This behavior is formulated by Barankin bound [15]. An alternative definition of SNR threshold is developed from the results in Fig. 4, which is the SNR point where the noise effects become negligible. As a result, bandwidth effects also begin to contribute to the overall MSE performance. In other words, SNR is the dominant parameter in the low SNR region and both SNR and $B$ contributes to the overall MSE performance after the SNR threshold. This definition is also useful to explain the performance gap between ML and CRLB in the low SNR (or nonlinear) region. This is because of CRLB takes both SNR and $B$ into account during time delay estimate over the entire SNR region. Therefore, CRLB performs better than ML in the low SNR region and ML converges to the approximate CRLB after the SNR threshold. Moreover, ML performs better than the approximate CRLB by $0.5 \mathrm{~dB}$ according to the results in Fig. 4 .

Performance of whole and dispersed spectrum utilization systems are compared considering approximate CRLB and ML. Performance comparison of both approaches are conducted for three cases. Note that the signals from all branches are combined in dipersed spectrum utilization method. The number of available dispersed bandwidth for all three cases is considered to be 2, i.e. $K=2$. In case $1, S N R_{1}+$ $S N R_{2}=S N R, B_{i}=1 \mathrm{MHz}$ and $B=2 \mathrm{MHz}$. For case 2, $S N R_{i}=S N R, B_{i}=B=2 \mathrm{MHz}$. Finally, the parameters for case 3 are given as follows; $S N R_{1}+S N R_{2}=S N R$, $B_{i}=B=2 \mathrm{MHz}$. The remaining system parameters for all three cases are common and they are given as follows. Training data $d_{i, l}=d_{l}=1$ is considered, where the number of observation symbols for both whole and dispersed spectrum is 1, i.e. $N_{i}=N=1$. In addition, it is assumed that the spectral density of the noise is the same for the whole spectrum and all the $K$ branches of the dispersed spectrum techniques; i.e., $\sigma_{i}=\sigma$ for $i=1, \ldots, K$. The Gaussian second order derivative pulse shape is used. Therefore, the pulse shape for the whole and dispersed spectrum methods are generated using

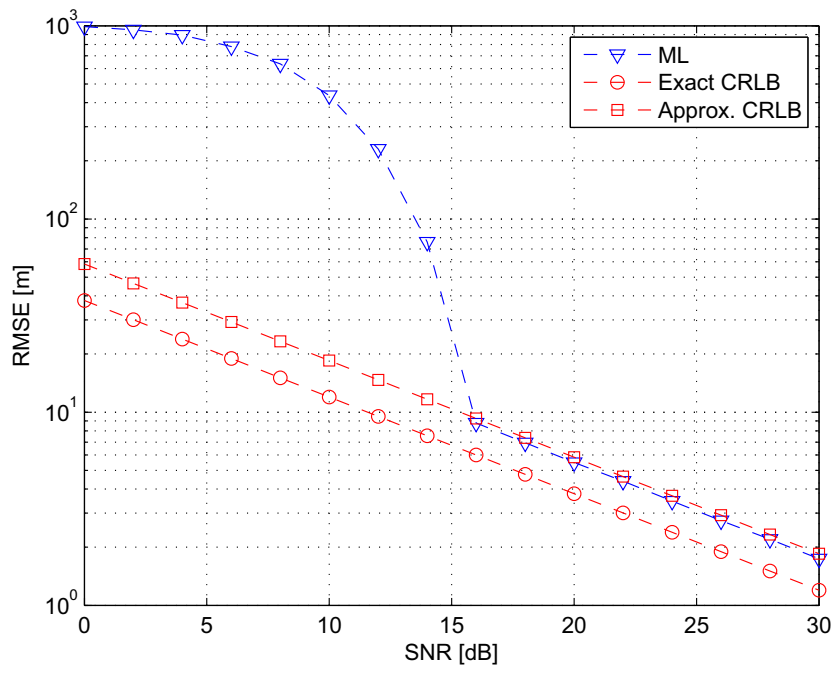

Fig. 4. Comparison of exact and approximate CRLB for whole spectrum utilization systems

$T_{p}=2.5 \zeta$ where $\zeta=1 / B$, and $T_{p i}=2.5 \zeta_{i}$ where $\zeta_{i}=1 / B_{i}$, respectively. Furthermore, the root mean square error (RMSE) metric is considered to measure the performance of ML time delay estimate. Note that all the results in this section are obtained over 10000 channel realizations. For the theoretical case, (7) is denoted as CRLB-Whole and (11) is denoted as CRLB-Dispersed. Similarly, ML time delay estimator for the whole (5) and dispersed spectrum (9) cases are denoted as ML-Whole and ML-Dispersed, respectively. The results for case 1 are plotted in Fig. 5. CRLB-Whole performs better than CRLB-Dispersed by $6 \mathrm{~dB}$ for the entire SNR region. Since the total SNR for dispersed case is equal to $S N R$,

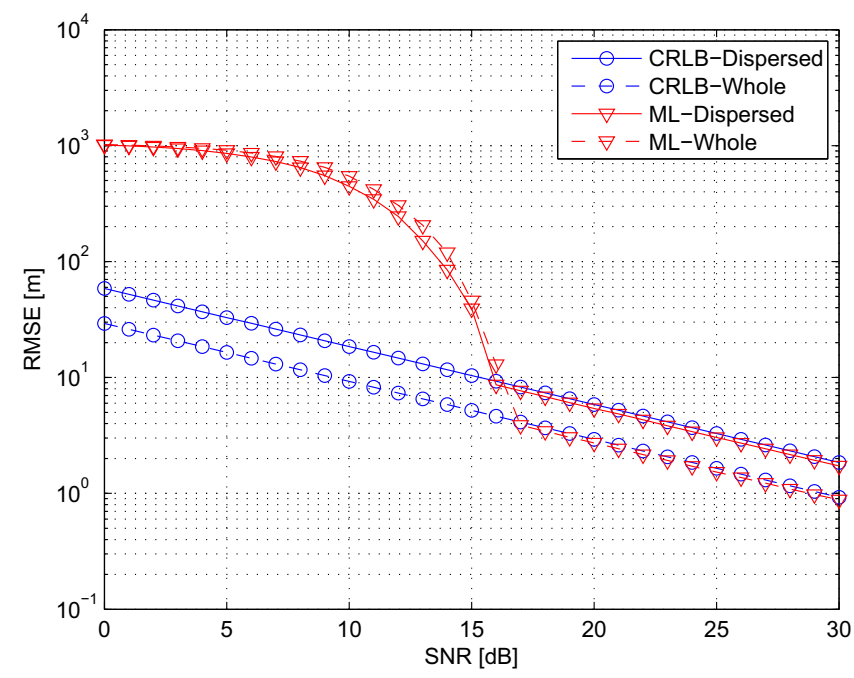

Fig. 5. ML and $\sqrt{\mathrm{CRLB}}$ versus $\mathrm{SNR}$ for dispersed and whole spectrum utilization. 


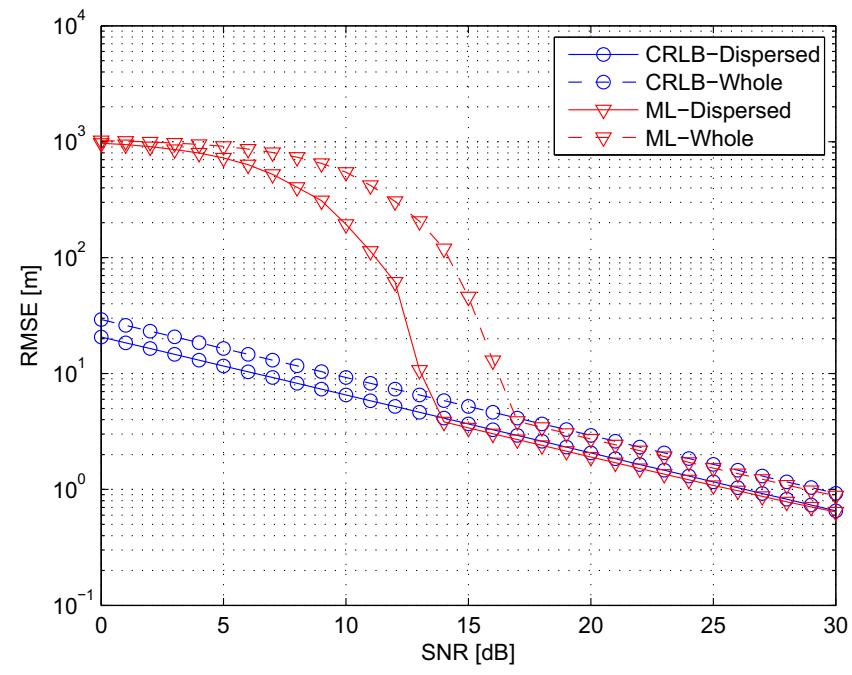

Fig. 6. $\mathrm{ML}$ and $\sqrt{\mathrm{CRLB}}$ versus SNR for dispersed and whole spectrum utilization.

this performance difference is due to $B^{2}=4 B_{i}^{2}$. This result is justified by the first condition in (13). On the other hand, ML-Whole performs similar to ML-Dispersed in the low SNR (nonlinear) region since the total SNR for dispersed case is equal to $S N R$. However, in the linear region (after the SNR threshold of $17 \mathrm{~dB}$ ), ML-Whole and ML-Dispersed converges to CRLB-Whole and CRLB-Dispersed, respectively. In other words, ML-Whole performs better than ML-Dispersed by $6 \mathrm{~dB}$ in this region. Furthermore, the SNR threshold for dispersed is less than the SNR threshold for whole case and this is due to SNR gain obtained in the dispersed spectrum utilization case. The results for case 2 are plotted in Fig. 6. According

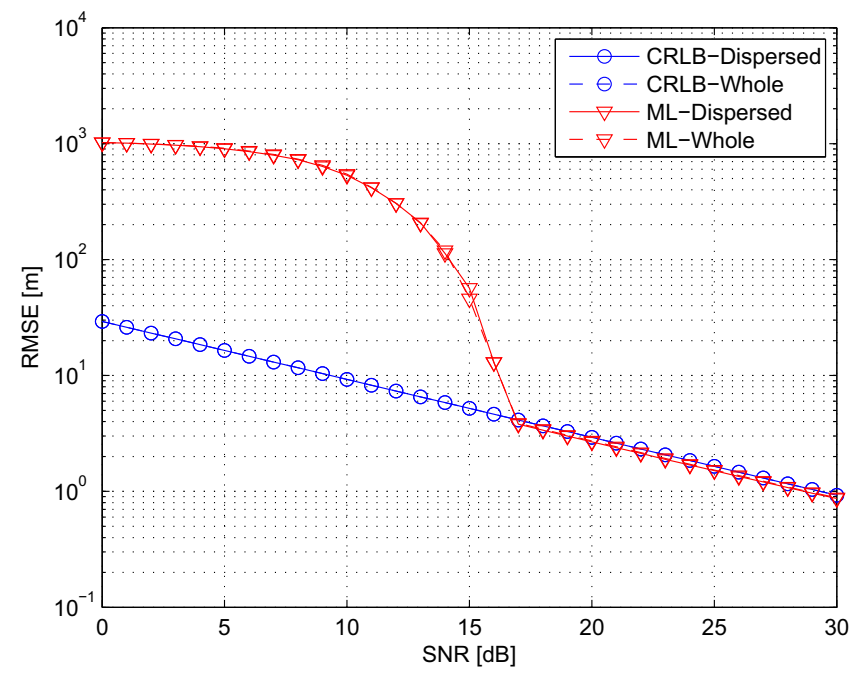

Fig. 7. $\mathrm{ML}$ and $\sqrt{\mathrm{CRLB}}$ versus SNR for dispersed and whole spectrum utilization. to the results, CRLB-Dispersed performs better than CRLBwhole by $3 \mathrm{~dB}$ for the entire SNR region. This is due to SNR gain obtained in the dispersed case since $B_{i}=B=2 \mathrm{MHz}$. This result is justified with the second condition in (13). On the other hand, ML-Dispersed performs better than ML-Whole by $3 \mathrm{~dB}$ in the low SNR region due to the SNR gain. The same result is observed in the linear region since $B_{i}=B$. In other words, bandwidth does not cause any performance difference in this case.

In Fig. 7, the results for case 3 are shown. CRLB-Whole and CRLB-Dispersed performs same for all the SNR values. This result is justified by the third condition in (13). Similarly, MLWhole and ML-Dispersed performs same over all SNR region since the total SNR in dispersed case is equal to $S N R$ and $B_{i}=B$.

\section{CONCLUSIONS}

In this paper, performance of whole and dispersed spectrum utilization approaches are compared considering time delay estimation problem in cognitive radio systems. The performance of both approaches are compared theoretically based on CRLB and ML time delay estimation method. The results show that dispersed spectrum utilization method performs equivalent to whole spectrum utilization method under the condition that the SNR at each branch is combined when the bandwidth of signal at each branch is equal. This indicate that the performance of dispersed spectrum utilization method depends on the combining technique, which can be investigated further. In addition, it is observed that conventional whole spectrum utilization method is special case of dispersed spectrum utilization method. In other words, dispersed spectrum utilization is a method that exploits frequency diversity in wireless systems in full extent, which can provide much more flexibility to adapt the range accuracy in cognitive radio systems.

\section{ACKNOWLEDGEMENTS}

The authors wish to thank Dr. Sinan Gezici for his fruitful discussions and comments. Furthermore, we wish to acknowledge the support of Qatar Foundation for Education, Science, and Community Development and Qatar Telecom (Qtel) during this work.

\section{REFERENCES}

[1] J. Mitola and G. Q. Maguire, "Cognitive radio: Making software radios more personal," IEEE Personal Commun. Mag., vol. 6, no. 4, pp. 13-18, August 1999

[2] S. Haykin, "Cognitive Radio: Brain-empowered Wireless Communications," IEEE J. Select. Areas Commun., vol. 23, no. 2, pp. 201-220, Feb. 2005.

[3] Y. Xing and R. Chandramouli and S. Mangold and S. Shankar N, "Dynamic Spectrum Access in Open Spectrum Wireless Networks," IEEE J. Select. Areas Commun., vol. 24, no. 3, pp. 626-637, March 2006.

[4] H. Celebi and H. Arslan, "Ranging Accuracy in Dynamic Spectrum Access Networks," IEEE Commun. Lett., vol. 11, no. 5, pp. 405-407, May 2007.

[5] _ " "Enabling location and environment awareness in cognitive radios," Elsevier Computer Communications-Special Issue on Advanced Location-Based Services, vol. 31, no. 6, pp. 1114-1125, Apr. 2008. 
[6] S. Gezici, H. Celebi, H. Vincent Poor and H. Arslan, "Fundamental limits on time delay estimation in cognitive radio systems," IEEE Trans. on Wireless Communications, vol. 8, no. 1, pp. 78-83, Jan. 2009.

[7] S. Gezici, H. Celebi, H. Arslan, and H. V. Poor, "Theoretical limits on time delay estimation for ultra-wideband cognitive radios," in IEEE International Conference on UWB (ICUWB), vol. 2, Hannover, Germany, Sept. 2008, pp. 177-180.

[8] R. I. C. Chiang, G. B. Rowe, and K. W. Sowerby, "A quantitative analysis of spectral occupancy measurements for cognitive radio," in Proc. IEEE IEEE Vehicular Technology Conference, Dublin, Ireland, Apr. 2007.

[9] T. A. Weiss and F. K. Jondral, "Spectrum pooling: An innovative strategy for the enhancement of spectrum efficiency," IEEE Commun. Mag., vol. 42, no. 3, pp. 8-14, March 2004.

[10] S. Brandes, I. Cosovic, and M. Schnell, "Sidelobe suppression in ofdm systems by insertion of cancellation carriers," in Proc. IEEE Veh. Technol. Conf., vol. 1, Dallas, TX, USA, Sep. 2005, pp. 152-156.

[11] _ - "Reduction of out-of-band radiation in OFDM based overlay systems," IEEE Commun. Lett., vol. 10, no. 6, pp. 420-422, June 2006.

[12] H. Mahmoud and H. Arslan, "Spectrum shaping of ofdm-based cognitive radio signals," in Proc. IEEE Radio and Wireless Symposium, Orlando, FL, USA, Jan. 2008.

[13] H. Celebi and H. Arslan, "Cognitive Positioning Systems," IEEE Trans. Wireless Commun., vol. 6, no. 12, pp. 4475-4483, Dec. 2007.

[14] H. Celebi, "Location awareness in cognitive radio networks," Ph.D. dissertation, University of South Florida, FL, Aug. 2008.

[15] A. Zeira and P. Schultheiss, "Realizable lower bounds for time delay estimation," IEEE Trans. on Signal Processing, vol. 41, no. 11, pp. 3102-3113, Nov. 1993. 